\title{
Archivos

\section{El síndrome de QT largo: una breve revisión del diagnóstico electrocardiográfico incluyendo la prueba de Viskin ${ }^{\text {is }}$}

\author{
Manlio F. Márquez* \\ Departamento de Electrocardiología, Instituto Nacional de Cardiología Ignacio Chávez, México D.F., México
}

Recibido el 7 de junio de 2012; aceptado el 8 de junio de 2012

\section{PALABRAS CLAVE \\ Síndrome de QT \\ largo; \\ Muerte súbita; \\ Electrocardiograma; \\ Prueba de Viskin; \\ México}

Resumen El intervalo QT mide tanto la repolarización como la despolarización. Aprender a medir el intervalo QT y saber corregirlo (QTc) para la frecuencia cardiaca (FC) es básico, pues permite establecer el diagnóstico de un síndrome de QT largo (SQTL). El intervalo QTc puede variar en duración e incluso morfología, dependiendo de la hora y del día. Existe una respuesta adaptativa disminuida del intervalo QTC a los cambios en la FC, que se conoce como histéresis del QT. Viskin ha introducido una prueba clínica bastante sencilla para confirmar el diagnóstico de SQTL, que se basa en la "hipoadaptación" del intervalo QT al ponerse de pie, y que en el electrocardiograma (ECG) de superficie da la apariencia de un "estiramiento del QT"'. Asimismo, ha acuñado el término de "aturdimiento del QT" para hacer referencia al fenómeno de que el intervalo QTC no regresa al valor inicial basal, a pesar de recuperarse la FC basal después del ortostatismo. En este artículo se muestran algunos ejemplos y la manera de realizar la prueba de Viskin.

(c) 2012 Instituto Nacional de Cardiología Ignacio Chávez. Publicado por Masson Doyma México S.A. Todos los derechos reservados.

Long QT syndrome: a brief review of the electrocardiographical diagnosis including Viskin's test

Abstract The QT interval measures both repolarization and depolarization. Learning to measure the QT interval and know how to correct (QTc) for heart rate (HR) is essential for the diagnosis of long QT syndrome (LQTS). The QTC interval changes in duration and even morphology depending on the time of the day and on a day-to-day basis. A diminished adaptive response of the QTc interval to changes in HR is known as QT hysteresis. Viskin has introduced a very simple clinical test to confirm the diagnosis of LQTS based on the "hypoadaptation" of the QT when standing. This phenomenon gives the appearance of a "stretching of the QT" on

\footnotetext{
Ponencia presentada en Venice Arrhythmias, Octubre 2011 y $2^{a}$ Sesión Estatutaria de la Sociedad Mexicana de Cardiología, Junio 2012. * Autor para correspondencia: Instituto Nacional de Cardiología Ignacio Chávez. Juan Badiano 1, Colonia Sección XVI, Tlalpan. C.P. 14080. México D.F., México. Teléfono: +52 (55) 55732911.

Correo electrónico: manlio.marquez@gmail.com
} 
the surface ECG. Likewise, he has coined the term "QT stunning" to refer to the phenomenon that the QTc interval does not return to baseline despite recovery of baseline HR after standing. This article shows some examples of the Viskin's test.

(C) 2012 Instituto Nacional de Cardiología Ignacio Chávez. Published by Masson Doyma México S.A. All rights reserved.

\section{Antecedentes}

El síndrome de QT largo (STQL) es uno de los síndromes hereditarios más frecuentes asociados a muerte súbita ${ }^{1}$ (fig. 1). Se ha denominado como una channelopathy en inglés, lo que se ha traducido como una "canalopatía", aunque el término en español suena demasiado fuerte y en lo personal, prefiero usar "enfermedad de canales iónicos". Sabemos que el potencial de acción cardíaco depende de un delicado equilibrio entre las corrientes iónicas de despolarización y repolarización, las cuales se producen gracias al correcto funcionamiento de los correspondientes canales iónicos. De ahí que una alteración en los genes que codifican para dichos canales, se vea traducida en un desequilibrio en dichas corrientes. La repolarización ventricular normal se produce por el equilibro entre las corrientes de entrada de sodio y calcio, y la corrientes de salida de potasio $(\mathrm{K}+)$. Éstas últimas son varias pero se pueden agrupar en dos, aquellas encargadas de la repolarización en la fase inicial del potencial de acción, que se denominan "corrientes hacia afuera" (transient outward en inglés; $I_{\mathrm{TO}}$ ) y aquellas encargadas de resto de la repolarización que se conocen como corrientes "rectificadoras tardías" ( $\left(I_{\mathrm{kr}}, I_{\mathrm{ks}}, I_{\mathrm{kur}}\right)$. Existen diversas mutaciones que pueden afectar a los canales iónicos. Los efectos de dichas mutaciones se pueden agrupar en tres: afección de la permeabilidad del canal, alteración de la activación del canal y disfunción en la inactivación del canal.

\section{El intervalo QT}

Desde el punto de vista electrocardiográfico, la repolarización ventricular normal está representada por el segmento ST y la onda T. Sin embargo, el parámetro electrocardiográfico con el que contamos para valorar la duración de la repolarización es el intervalo QT. Es importante recordar que dicho intervalo QT comprende no sólo a la repolarización, sino también a la despolarización ya que incluye al complejo QRS. Este hecho es fundamental para comprender porque se puede alargar en presencia de trastornos de la conducción, y no sólo por alteraciones de la repolarización. En el caso que nos ocupa, el electrocardiograma (ECG) del STQL se caracteriza por una alteración en la repolarización, la cual es anormalmente larga. Tomando la premisa antes mencionada del equilibrio entre corrientes de entrada y salida, un SQTL se puede producir por alteraciones en los canales de $\mathrm{K}+$, que consecuentemente disminuyen la corriente de salida de $\mathrm{K}+$. También se puede producir, si alteraciones del canal de sodio o calcio aumentan en forma anormal la entrada a la célula de cualquiera de estos iones. En cualquier caso, la repolarización tan prolongada puede resultar, generalmente favorecida por ciertos factores desencadenantes o precipitantes, en un tipo específico de taquicardia ventricular polimórfica cuya morfología característica refleja el torsal usado durante el tejido, motivo por el cual el Dr. Dessertennes bautizó como torsades des pointes, que corresponderia en español a "tejido torsal" y en México el grupo del Maestro Cárdenas tradujo como "taquicardia helicoidal",2.

\section{Tipos y subtipos de SQTL}

Aunque se conocen en la actualidad 13 síndromes de QT largo, en realidad los más importantes, principalmente por su elevada frecuencia son los denominados tipos 1 a $3^{3}$. Se estima que un $90 \%$ de los casos de SQTL que tienen diagnóstico genético, presentan alguno de estos tres. Sin embargo, siempre es importante resaltar los siguientes hechos respecto al SQTL: 1) una de las pocas familias a nivel mundial y probablemente una de las más largas con síndrome de Andersen (SQTL7) es mexicana ${ }^{4}, 2$ ) fue con una familia mexicana que se describió el SQTL10 ${ }^{5}$, y 3 ) uno de los raros casos de síndromes de sobreposición (overlapping) fue descrito en un paciente mexicano ${ }^{6}$. Dicho lo anterior, regresemos a los tipos más frecuentes. Se sabe que existe hasta cierto punto una estrecha correlación entre el genotipo (SQTL 1, 2 o 3) y el fenotipo (su expresión clínica). Se han identificado diferencias importantes entre cada uno de estos SQTL en relación a su manifestación electrocardiográfica, evolución clínica, factores disparadores, respuesta a la estimulación simpática y respuesta a beta-bloqueadores. Los ECGs representativos de cada uno de estos SQTL fueron descritos originalmente por Moss y colaboradores en 1995. Destacaban como características específicas, la presencia en el SQTL1 de una onda T de gran tamaño e inicio "temprano"' (fig. 1), mientras que en el SQTL2 la onda T es de inicio un poco más "tardío"' y no de gran tamaño, pero si puede tener muescas (onda $T$ mellada). En cambio, la morfología característica del SQTL3 es una onda $\mathrm{T}$ de inicio muy tardío y generalmente acuminada y de base corta.

\section{Dinámica del QT}

El intervalo QT guarda una relación directa con el intervalo $\mathrm{RR}^{7}$. A raíz de este hecho, se han diseñado varias fórmulas matemáticas para "corregir" el intervalo QT y adecuarlo a la frecuencia cardiaca o lo que es lo mismo, al intervalo RR. Por ello, el intervalo QT clínicamente útil es el intervalo QT corregido (QTC). Aunque existen varias fórmulas para 


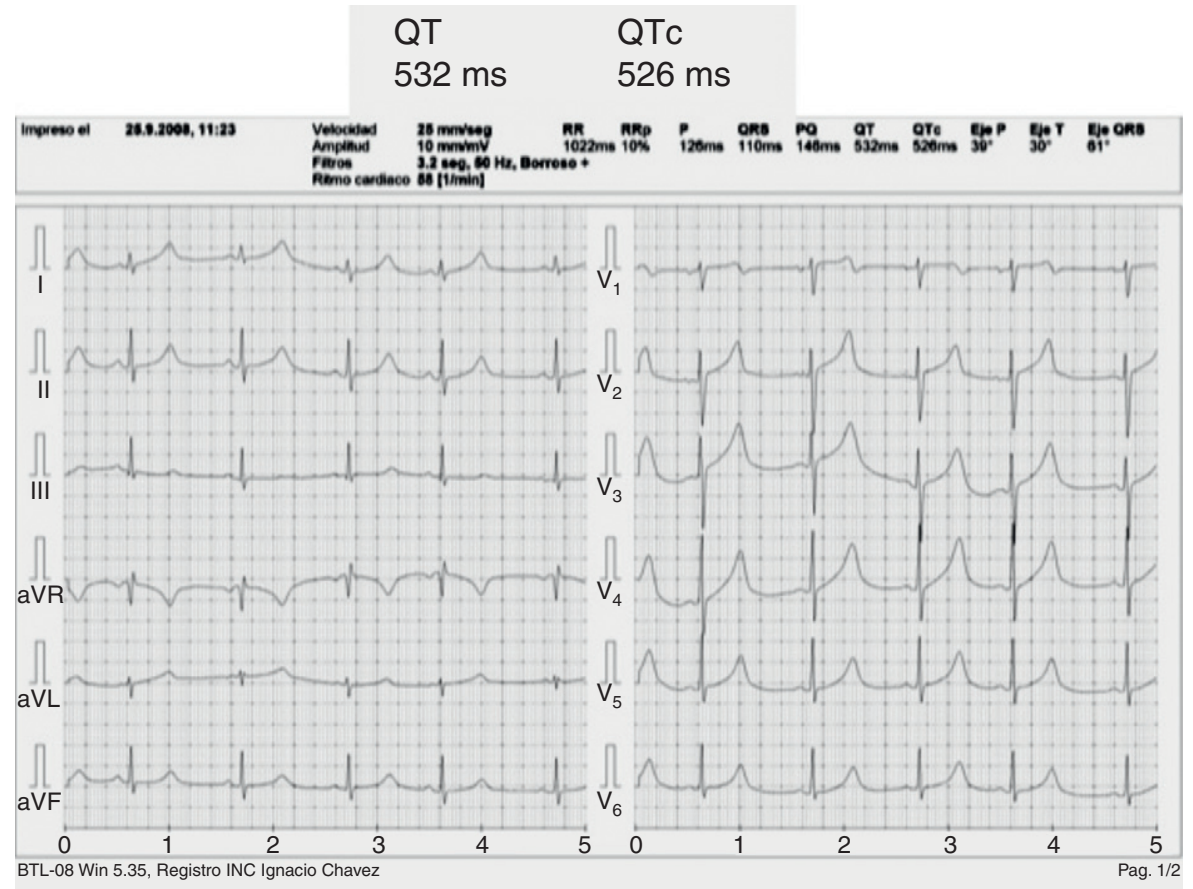

Figura 1 Electrocardiograma de 12 derivaciones de una paciente con SQTL. El intervalo QT mide 532 ms y el QTc 526 ms. Nótese la morfología en V5 característica del SQTL tipo 1.

corregir el QT, la más empleada es la de Bazett descrita en $1920^{8}$, como QT $=\mathrm{K} \times \mathrm{RR}^{0.5}$ y modificada en 1947 por Taran y Szilagyi, a la fórmula actualmente empleada en clínica: $\mathrm{QTC}=\mathrm{QT} / \sqrt{ }$ RR. Nótese que para obtener un resultado válido, el intervalo RR debe estar en segundos. Uno de los errores más comunes en el cálculo del intervalo QTc, radica en que no se calcula la raíz del RR en segundos sino en milisegundos, por lo que se obtienen resultados incorrectos. Otros errores pueden ser atribuidos a la falta de experiencia para medir el intervalo QT o a la dificultad para definir el final de la
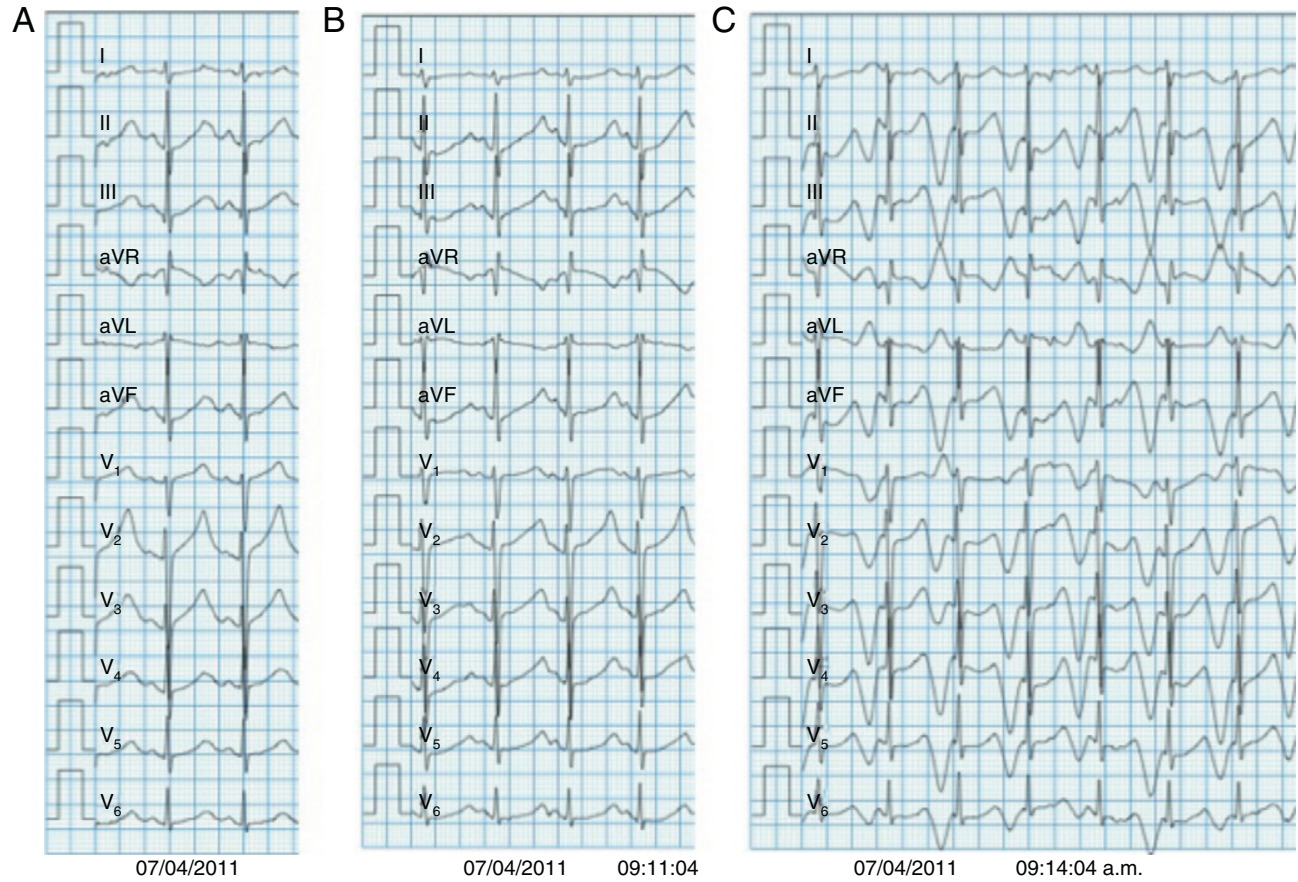

Figura 2 Electrocardiogramas representativos durante la prueba de esfuerzo de la misma paciente presentada en la figura 1. A) Inicio de ejercicio. B) Durante el esfuerzo, nótese como el final del intervalo QT se pega al inicio de la onda P, simulando un "estiramiento del QT"'. C) Al máximo esfuerzo, se observa alternancia de la onda T (signo de mal pronóstico). 
onda $\mathrm{T}^{9}$. Por ello, es importante que el cardiólogo conozca estos detalles para obtener QTc válidos. Sin embargo, ésta tiende a sobreestimar el QT a frecuencias altas y subestimarlo a frecuencias bajas. Para evitar esto se pueden usar las fórmulas de Friedericia, Hodges o Framingham ${ }^{10-12}$.

Por otro lado, el intervalo QT no sólo depende del intervalo RR precedente sino que se debe adaptar a los cambios en la frecuencia cardiaca $(F C)^{13}$. Al hecho de que normalmente el intervalo QT se retrasa un poco para adaptarse a los incrementos de la FC, se le conoce como "histéresis" del QT o "retraso" del QT (QT lag). Si se grafican los intervalos RR y QT en forma simultánea, se puede observar claramente dicho retraso. Se sabe que los intervalos QT pueden variar día con día, en un mismo día de acuerdo al ritmo circadiano y en relación con la edad ${ }^{14}$.

\section{La prueba de Viskin: el estiramiento del QT y el aturdimiento del QT}

Estudiando la histéresis del QT en los SQTL, diversos investigadores observaron que los pacientes con SQTL presentaban una histéresis anormalmente prolongada con el ortostatismo y el ejercicio ${ }^{15}$. En la figura 2 se observan los ECGs representativos durante una prueba de esfuerzo, pertenecientes a una paciente con SQTL1 ${ }^{16}$. Se puede apreciar el alargamiento del intervalo QTc con el ejercicio, e incluso la aparición de alternancia de la onda $\mathrm{T}$ al final del ejercicio. Viskin por su parte introdujo el término de estiramiento del QT (QT stretching) para hacer referencia al fenómeno, que ocurre cuando el intervalo QT no se acorta en forma proporcional al acortamiento del intervalo RR (cuando aumenta la $\mathrm{FC})^{17}$. Esto produce la imagen de un QT muy largo, en comparación con el RR, dando la impresión de que se estira, de ahí el nombre. Más importante es la medición del intervalo QTc. En la figura 3 se observan los trazos comparativos de una paciente con SQTL. El trazo basal muestra un QT de $560 \mathrm{~ms}$ para un QTC de $601 \mathrm{~ms}$ (FC 69 (pm), mientras que al adoptar el ortostatismo, el QT se acorta a $520 \mathrm{~ms}$ pero debido a que la FC se incrementa a $99 \mathrm{lpm}$, el QTc es de $668 \mathrm{~ms}$. La sencillez de esta prueba ha hecho que en poco tiempo se conozca ya como la prueba de Viskin, siendo especialmente útil para los casos de QT limítrofe (entre 440 y $480 \mathrm{~ms}$ ). En casos con SQTL franco, la simple adopción de la posición de pie puede incluso producir extrasístoles o
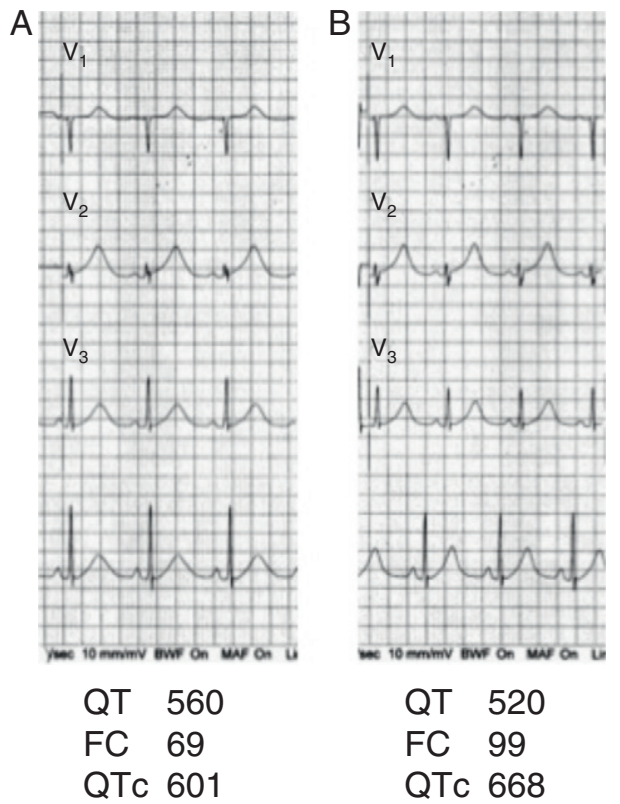

Figura 3 Derivaciones V1 a V3 de un paciente con SQTL. En el trazo basal el QTc es de $601 \mathrm{~ms}$, al adoptar el ortostatismo la FC se incrementa a $99 \mathrm{lpm}$ y el QTc a $668 \mathrm{~ms}$.

alteraciones significativas de la repolarización, tal cual como ocurre con el ejercicio. Un incremento mayor de $50 \mathrm{~ms}$ del QTc con la máxima FC o el máximo estiramiento del QT tiene una sensibilidad del $90 \%$ y una especificidad del $86 \%$, respectivamente, para diagnosticar SQTL en el grupo de población con QTc entre 390 y 480 ms basales. Estudios han mostrado que este fenómeno se reduce sustancialmente con el uso de beta-bloqueadores, por lo cual también podría servir para monitoreo del tratamiento ${ }^{18}$.

Por otro lado, la capacidad de recuperación del intervalo QTc después del ejercicio también se ve afectada en los individuos con SQTL. Viskin introdujo el término de "aturdimiento del QT" (QT stunning) para referirse al hecho de que el QTc medido cuando el sujeto regresa a la FC basal después de ponerse de pie, es más largo que el QTc basal a la misma $\mathrm{FC}^{19}$. Un diagrama de cómo realizar la prueba de Viskin se puede observar en la figura 4.

\section{Prueba de Viskin}

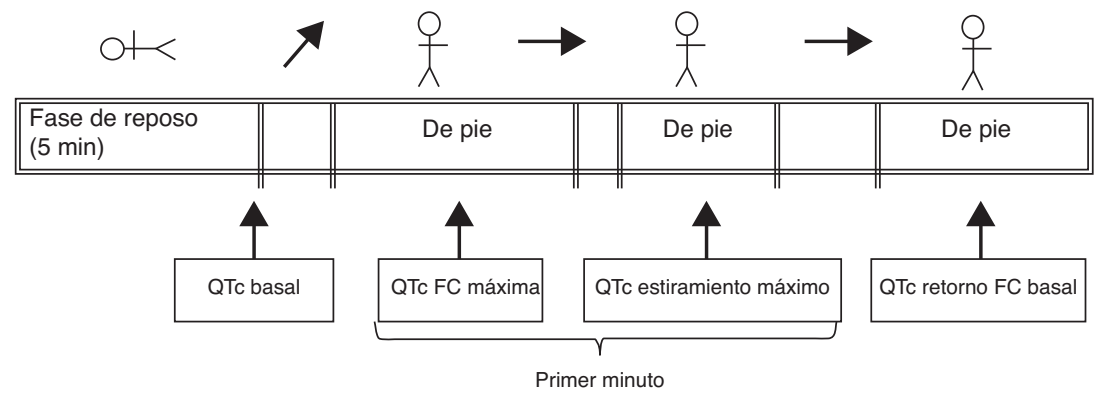

Figura 4 Gráfico que ejemplifica la manera de realizar la prueba de Viskin. 


\section{Conclusión}

Existe una respuesta adaptativa disminuida del intervalo QTC a los cambios en la FC, que se conoce como "histéresis del QT' ' Viskin ha introducido dos pruebas clínicas bastante sencillas, que se basan en dicha "hipoadaptación" del intervalo QT al ponerse de pie, las cuales pueden ser útiles para confirmar el diagnóstico de SQTL.

\section{Financiamiento}

No se recibió patrocinio de ningún tipo para llevar a cabo este artículo.

\section{Conflicto de intereses}

El autor declara no tener ningún conflicto de intereses.

\section{Referencias}

1. Iturralde-Torres $P$, Medeiros-Domingo A. Genética en los síndromes de QT prolongado. Arch Cardiol Mex 2009;79 Suppl. 2:26-30.

2. Hermosillo JA, Cárdenas M, Hurtado L, et al. Taquicardia ventricular helicoidal (Torsades de Pointes). Arch Inst Cardiol Mex 1977;47:5-18.

3. Monteforte N, Napolitano C, Priori SG. Genética y arritmias: aplicaciones diagnósticas y pronósticas. Rev Esp Cardiol 2012;65:278-86.

4. Canún S, Pérez N, Beirana LG. Andersen syndrome autosomal dominant in three generations. Am J Med Genet 1999;85:147-56.

5. Medeiros-Domingo A, Kaku T, Tester DJ, et al. SCN4B-encoded sodium channel beta4 subunit in congenital long-QT syndrome. Circulation 2007;116:134-42.

6. Márquez MF, Cruz-Robles D, Inés-Real S, et al. A novel SCN5A deletion mutation in a child with ventricular tachycardia, recurrent aborted sudden death, and Brugada electrocardiographic pattern. Arch Cardiol Mex 2007;77:284-7.
7. Malik M, Hnatkova K, Novotny T, et al. Subject-specific profiles of QT/RR hysteresis. Am J Physiol Heart Circ Physiol 2008;295:H2356-63.

8. Bazett HC. An analysis of the time-relations of electrocardiograms. Heart 1920;7:35-70.

9. Viskin S, Rosovski U, Sands AJ, et al. Inaccurate electrocardiographic interpretation of long QT: the majority of physicians cannot recognize a long QT when they see one. Heart Rhythm 2005;2:569-74.

10. Fridericia LS. Die Systolendauer im Elekrokardiogramm bei normalen Menschen und bei Herzkranken. Acta Med Scand 1920;53:469-86.

11. Hodges M. Rate correction of the QT interval. Card Electrophysiol Rev 1997;1:360-3.

12. Sagie A, Larson MG, Goldberg RJ, et al. An improved method for adjusting the QT interval for heart rate (the Framingham Heart Study.). Am J Cardiol 1992;70:797-801.

13. Sredniawa B, Musialik-Lydka A, Jarski P, et al. Methods of assessment and clinical relevance of QT dynamics. Indian Pacing Electrophysiol J 2005;5:221-32.

14. Extramiana F, Maison-Blanche P, Badilini F, et al. Circadian modulation of QT rate dependence in healthy volunteers: gender and age differences. J Electrocardiol 1999;32: 33-43.

15. Wong JA, Gula LJ, Klein GJ, et al. Utility of treadmill testing in identification and genotype prediction in long-QT syndrome. Circ Arrhythm Electrophysiol 2010;3:120-5.

16. Márquez MF, Ramos-Kuri M, Hernández-Pacheco G, et al. KCNQ 1 (KvLQT1) missense mutation causing congenital long QT syndrome (Jervell-Lange-Nielsen) in a Mexican family. Arch Cardiol Mex 2006;76:257-62.

17. Viskin S, Postema PG, Bhuiyan ZA, et al. The response of the QT interval to the brief tachycardia provoked by standing: a bedside test for diagnosing long QT syndrome. J Am Coll Cardiol 2010;55:1955-61.

18. Krahn AD, Yee R, Chauhan V, et al. Beta blockers normalize QT hysteresis in long QT syndrome. Am Heart J 2002;143: 528-34.

19. Adler A, van der Werf C, Postema PG, et al. The phenomenon of «QT stunning»: The abnormal QT prolongation provoked by standing persists even as the heart rate returns to normal in patients with long QT syndrome. Heart Rhythm 2012;9: 901-8. 